\title{
NUMERICAL SIMULATION FOR TSUNAMI-HEIGHT REDUCTION USING A VERY LARGE FLOATING STRUCTURE
}

\author{
Taro Kakinuma ${ }^{1}$, Tatsuya Nakahira ${ }^{2}$, Takatsugu Kamba ${ }^{3}$, \\ Takahiro Murakami ${ }^{4}$, and Keisuke Nakayama ${ }^{5}$
}

\begin{abstract}
The tsunami-height reduction using a very large floating structure, i.e., VLFS, is discussed, with the water waves, interacting with a floating thin-plate, simulated numerically. The final tsunami-height reduction rate increases, as VLFS length, VLFS flexural rigidity, or the wave height of an incident tsunami, is increased. If two VLFSs are utilized, the final tsunami-height reduction rate, also depends on the distance between the VLFSs. In two-dimensional tsunami propagation, another wave propagates to the outside, along the crest line of the main wave, leading to an additional tsunami-height reduction.
\end{abstract}

Keywords: VLFS; tsunami; wave-height reduction; thin-plate; floating-body wave

\section{INTRODUCTION}

A very large floating structure (VLFS), designed for an offshore airport, a storage facility, a wind/solar power plant, an emergency base, or others, has advantages including mobility, and also environment friendliness, for seawater can flow under the structure. The interaction between a flexible VLFS, and seawater, has been investigated through numerical calculation by e.g. Hermans (2000), and Kashiwagi (2004), in the cases concerning an airplane moving on a floating airport. Conversely, Sakai et al. (1998) performed hydraulic experiments, where a solitary wave traveled through a floating thinplate, showing wave disintegration, with a decrease in the wave height of the incident wave. This result suggests that the wave height of a huge tsunami decreases, owing to its propagation through an offshore VLFS. In the present study, we numerically simulate water waves, interacting with a floating thin-plate, to discuss the tsunami-height reduction using a VLFS.

\section{NUMERICAL METHOD}

The horizontal length of a structure, is assumed to be much larger than its thickness, resulting in a classical equation for an elastic thin-plate. We solve the set of governing equations (Kakinuma et al. 2012), based on a variational principle (Kakinuma 2001), for irrotational motion of inviscid, and incompressible, fluids, interacting with a thin-plate, floating at the water surface. In the derivation process of the governing equations, the fluid velocity potential $\phi$, is expanded into a power series of vertical position $z$, as

$$
\phi(\boldsymbol{x}, z, t)=\sum_{\alpha=0}^{N-1}\left(f_{\alpha}(\boldsymbol{x}, t) \cdot z^{\alpha}\right),
$$

where $N$ denotes the number of terms for the expanded velocity potential. In case $N$ is zero, we obtain a set of equations for the shallow water condition, with thin-plate flexural rigidity. The friction is neglected for simplicity.

The governing equations for surface waves, are transformed to finite difference equations, and solved using a rewritten version of the numerical program, which was developed by Nakayama and Kakinuma (2010) to simulate internal waves. The numerical results for the surface displacements, are in good agreement with the existing experimental data, obtained by Sakai et al. (1998), as shown in the model validation by Kakinuma et al. (2012).

Sketched in Fig. 1 is an example of a VLFS, with a tsunami propagating towards it. The flexural rigidity of a VLFS, is given in part of a numerical computation domain. In the present calculation, the still water depth equals $50.0 \mathrm{~m}$.

\footnotetext{
${ }^{1}$ Graduate School of Science and Engineering, Kagoshima University, 1-21-40 Korimoto, Kagoshima, Kagoshima 890-0065, Japan

${ }^{2}$ Department of Coastal Service, Echo Co. Ltd., 2-6-4 Kita-Ueno, Taito-ku, Tokyo 110-0014, Japan

${ }^{3}$ Department of Railroads, Daitetsu Kogyo Co., Ltd., 3-9-15 Nishinakajima, Yodogawa-ku, Osaka, Osaka 532-0011, Japan

${ }^{4}$ Division of System Science and Engineering, Kansai University, 3-3-35 Yamate-cho, Suita, Osaka 564-8680, Japan

${ }^{5}$ Graduate School of Engineering, Kobe University, 1-1 Rokkodai-cho, Nada-ku, Kobe, Hyogo 657-8501, Japan
} 


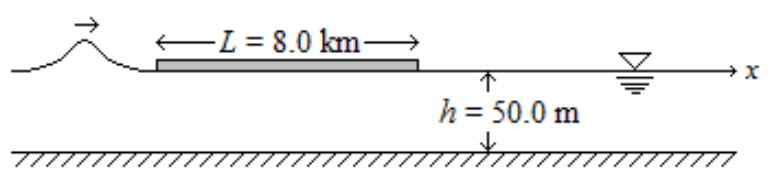

Figure 1. A sketch of an example of a VLFS, where the VLFS length $L$ is $8.0 \mathrm{~km}$, and the still water depth $h$ is $50.0 \mathrm{~m}$. A tsunami is traveling towards the VLFS.

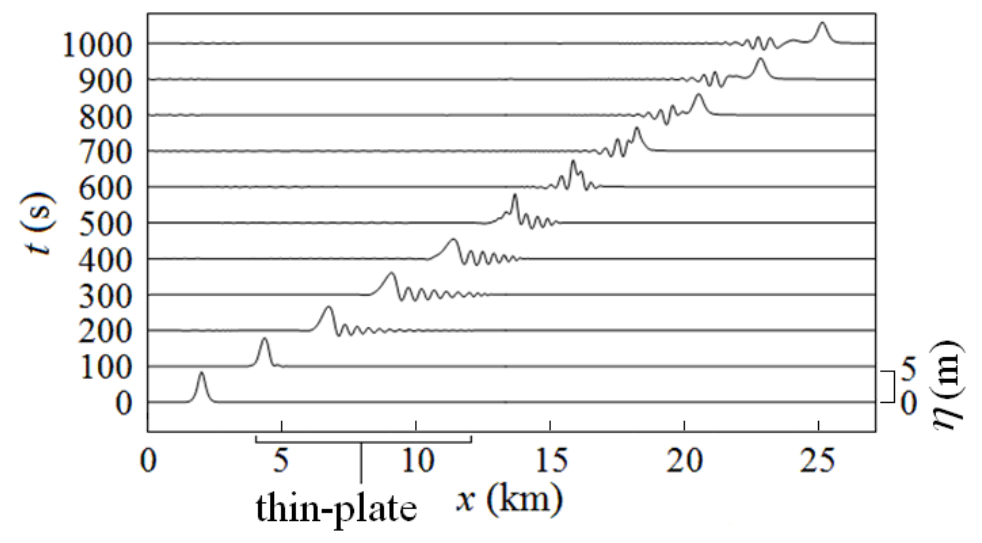

Figure 2. A numerical result of surface displacement $\eta$, where the VLFS flexural rigidity $B$ is $1.0 \times 10^{11} \mathrm{Nm}^{2}$, and the wave height of the incident wave, $a$, is $5.0 \mathrm{~m}$. The thin-plate covers the area where $4.0 \mathrm{~km} \leq x \leq 12.0$ $\mathrm{km}$. The still water depth is $50.0 \mathrm{~m}$.

\section{D TSUNAMI PROPAGATION THROUGH A VLFS}

A solitary wave solution, obtained using the numerical method developed by Yamashita and Kakinuma (2014), is given as an incident tsunami, where the above-described governing equations, are reduced only for stable water waves, without a thin-plate. The initial location of a wave peak, is $x=2.0$ $\mathrm{km}$ at $t=0.0 \mathrm{~s}$, while the offshore end of a VLFS, is located at $x=4.0 \mathrm{~km}$. The number of terms for the velocity potential expanded as Eq. (1), $N$, is three, to consider both the nonlinearity, and the dispersion, of waves.

Shown in Fig. 2 is the time variation of the surface profile, where $\eta$ denotes surface displacement, and the wave height of the incident tsunami, $a$, is $5.0 \mathrm{~m}$. The area where $4.0 \mathrm{~km} \leq x \leq 12.0 \mathrm{~km}$, is covered with a floating thin-plate, the flexural rigidity of which, $B$, is $1.0 \times 10^{11} \mathrm{Nm}^{2}$, in the $x-z$ plane. Figure 2 indicates that while the solitary wave propagates through the floating thin-plate, the wave shows disintegration, leading to a creation of shorter floating-body waves with larger phase velocities, such that the wave height of the main wave, i.e., the tsunami height, decreases. After passing the thinplate, the main wave overlaps the shorter waves, for the phase velocity of the main wave, is larger than that of the shorter waves in the uncovered area, such that the tsunami height increases, especially when $t$ $=500.0 \mathrm{~s}$, as shown in Fig. 2. However, as the main wave leaves the shorter waves behind, its wave height decreases, which means that this VLFS is applicable to decreasing the tsunami height.

Figure 3 shows the relative maximum surface levels $\eta_{1, \max } / \eta_{0, \max }$, for different values of VLFS length $L$, where $\eta_{1, \max }(x)$, and $\eta_{0, \max }(x)$, denote the maximum surface levels at each location, with, and without, a VLFS, respectively. The wave height of the incident tsunami, i.e., the initial tsunami height $a$ is $5.0 \mathrm{~m}$, and the VLFS flexural rigidity $B$ is $1.0 \times 10^{12} \mathrm{Nm}^{2}$. The final value of the tsunami-height reduction rate, $R$, increases, as the structure is longer, where the tsunami-height reduction rate is defined as $\left(\eta_{0, \max }-\eta_{1, \max }\right) / \eta_{0, \max }$. In the cases where the VLFS length $L$ is $8.0 \mathrm{~km}$, and $10.0 \mathrm{~km}$, the tsunami height finally becomes around $60 \%$ of the initial tsunami height, as shown in Fig. 3(b).

Indicated in Fig. 4 are the relative maximum surface levels $\eta_{1, \max } / \eta_{0, \max }$, for different values of initial tsunami height $a$, where the VLFS flexural rigidity $B$ is $1.0 \times 10^{12} \mathrm{Nm}^{2}$, and the VLFS length $L$ is $4.0 \mathrm{~km}$. The final value of the tsunami-height reduction rate, $R$, increases, as the initial tsunami height increases. In the case where the initial tsunami height $a$ is $15.0 \mathrm{~m}$, the tsunami height finally becomes less than $50 \%$ of the initial tsunami height.

Shown in Fig. 5 are the relative maximum surface levels $\eta_{1, \max } / \eta_{0, \max }$, for different values of VLFS flexural rigidity $B$, where the initial tsunami height $a$ is $5.0 \mathrm{~m}$, and the VLFS length $L$ is $4.0 \mathrm{~km}$. The 


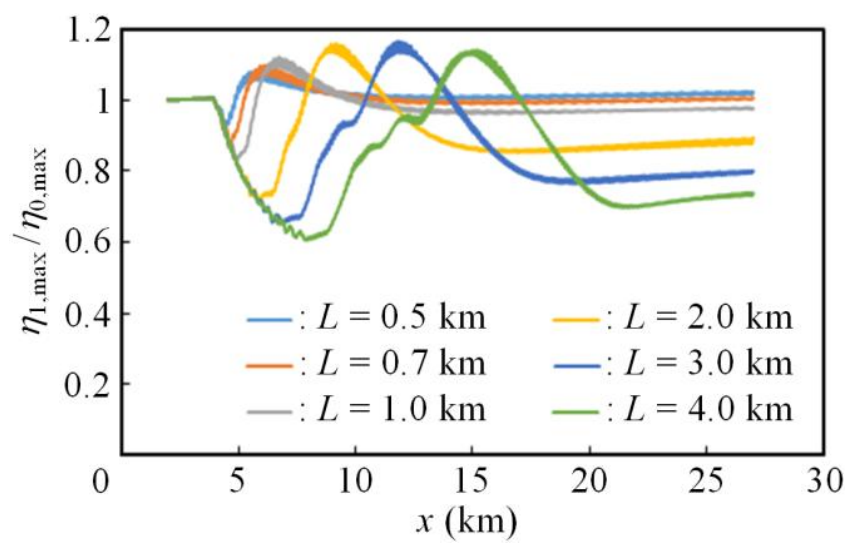

(a) $L=0.5 \mathrm{~km}, 0.7 \mathrm{~km}, 1.0 \mathrm{~km}, 2.0 \mathrm{~km}, 3.0 \mathrm{~km}$, and $4.0 \mathrm{~km}$

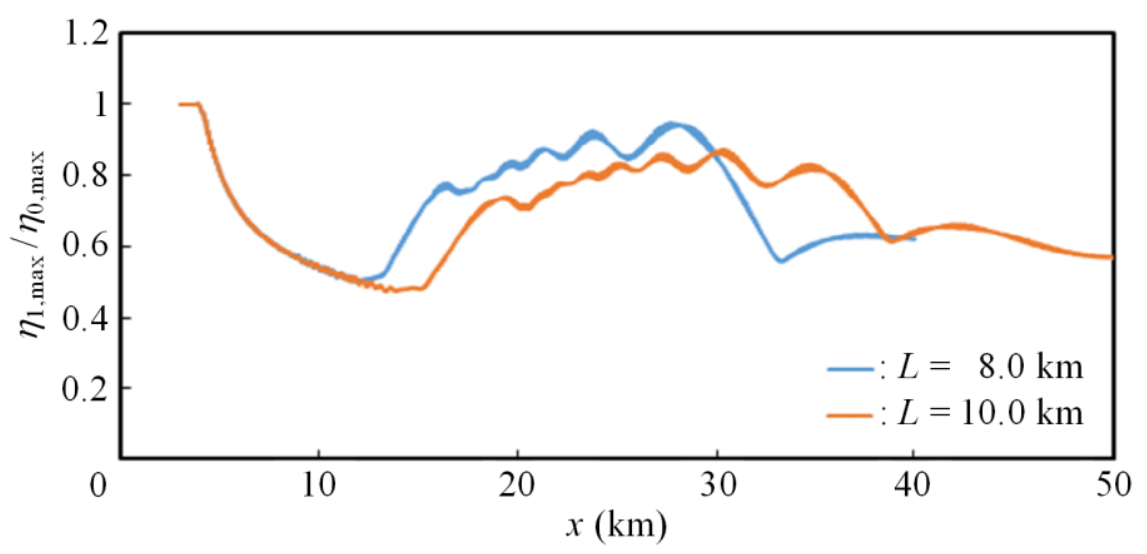

(b) $L=8.0 \mathrm{~km}$ and $10.0 \mathrm{~km}$

Figure 3. A relative maximum surface level $\eta_{1, \max } / \eta_{0, \max }$, for different values of VLFS length $L$, where the initial tsunami height $a$ is $5.0 \mathrm{~m}$. The VLFS flexural rigidity $B$ is $1.0 \times 10^{12} \mathrm{Nm}^{2}$, and the offshore end of the VLFS, is located at $x=4.0 \mathrm{~km}$. The still water depth is $50.0 \mathrm{~m}$.

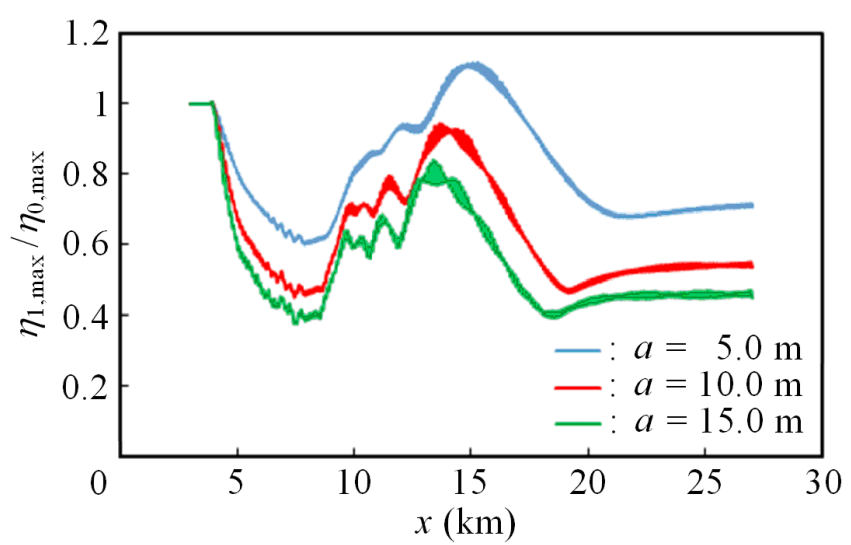

Figure 4. A relative maximum surface level $\eta_{1, \max } / \eta_{0, \max }$, for different values of initial tsunami height a, where the VLFS flexural rigidity $B$ is $1.0 \times 10^{12} \mathrm{Nm}^{2}$; the VLFS length $L$ is $4.0 \mathrm{~km}$; the offshore end of the VLFS, is located at $x=4.0 \mathrm{~km}$. The still water depth is $50.0 \mathrm{~m}$.

final value of the tsunami-height reduction rate, $R$, increases, as the VLFS flexural rigidity $B$ is increased. It should be noted that when $B$ is larger, it takes a longer time for the tsunami-height reduction rate, to reach its final value $R$, for the main wave, with a slower phase velocity due to a lower wave height, should pass a greater number of generated shorter waves. 


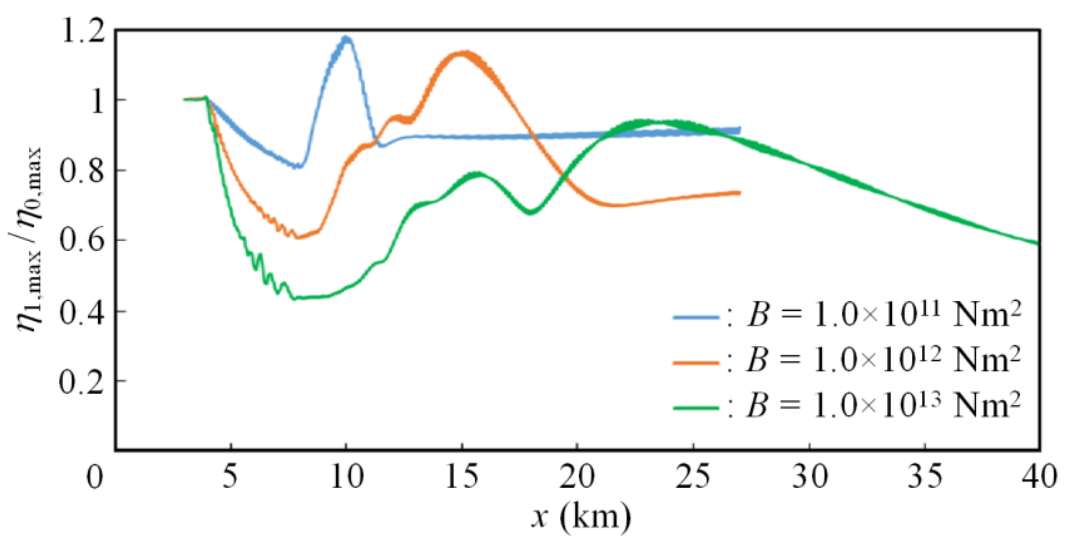

Figure 5. A relative maximum surface level $\eta_{1, \max } / \boldsymbol{\eta}_{0, \max }$, for different values of VLFS flexural rigidity $B$, where the initial tsunami height $a$ is $5.0 \mathrm{~m}$, and the VLFS length $L$ is $4.0 \mathrm{~km}$. The offshore end of the VLFS, is located at $x=4.0 \mathrm{~km}$. The still water depth is $50.0 \mathrm{~m}$.

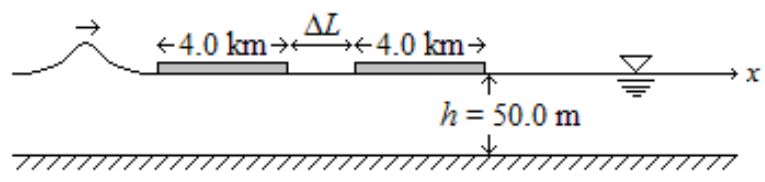

Figure 6. A sketch of an example of two VLFSs, where the length of both VLFSs is $4.0 \mathrm{~km}$, and $\Delta L$ denotes the distance between the VLFSs. The still water depth $h$ is $50.0 \mathrm{~m}$.

\section{D TSUNAMI PROPAGATION THROUGH TWO VLFS'S}

We study the tsunami height reduction using two VLFSs, as sketched in Fig. 6. In the present calculation, the flexural rigidity, and the length, of both VLFSs are $1.0 \times 10^{11} \mathrm{Nm}^{2}$, and $4.0 \mathrm{~km}$, respectively. The offshore end of the offshore-side VLFS, is located at $x=4.0 \mathrm{~km}$. A solitary wave solution, obtained using the numerical method developed by Yamashita and Kakinuma (2014), is given as an incident tsunami. The initial tsunami height $a$ is $5.0 \mathrm{~m}$, and the wave peak is initially located at $x=$ $2.0 \mathrm{~km}$ when $t=0.0 \mathrm{~s}$. The tsunami first attacks the offshore-side VLFS. The number of terms for the velocity potential expanded as Eq. (1), $N$, is three, to consider both the nonlinearity, and the dispersion, of waves.

Figure 7 shows the relative maximum surface levels $\eta_{1, \max } / \eta_{0, \max }$, for different values of the distance between the onshore end of the offshore-side VLFS, and the offshore end of the onshore-side VLFS, i.e., $\Delta L$. On the basis of the results, the final value of the tsunami-height reduction rate, $R$, depends on the distance between the VLFSs, $\Delta L$, since $R$ increases, as the wave height, as well as the wave steepness, of the main wave increases, when it enters the area covered with the onshore-side VLFS, where the wave height of the main wave increases, because of its overlap with generated shorter waves, as described above.

\section{D TSUNAMI PROPAGATION THROUGH A VLFS}

We also examine the two-dimensional propagation of a tsunami, interacting with a VLFS. Illustrated in Fig. 8 is the numerical computation domain, where a VLFS covers the area where $2.0 \mathrm{~km}$ $\leq x \leq 6.0 \mathrm{~km}$ and $0.0 \mathrm{~km} \leq y \leq 1.0 \mathrm{~km}$. The lateral boundary condition, along both $x=0.0 \mathrm{~km}$, and $y=$ $0.0 \mathrm{~km}$, is the reflection condition, while that along both $x=30.0 \mathrm{~km}$, and $y=2.0 \mathrm{~km}$, is the transmission condition. The VLFS area is $4.0 \mathrm{~km} \times 2.0 \mathrm{~km}$, owing to the mirror condition along the $x$ axis.

The initial surface profile is given by

$$
\eta(x, 0) / a=1+\cos [2 \pi(x / \lambda)] \quad \text { for } 0 \mathrm{~km} \leq x \leq \lambda / 2,
$$

where the initial wave height $a$, and the initial wavelength $\lambda$, of the incident tsunami are $5.0 \mathrm{~m}$, and 1.0 $\mathrm{km}$, respectively. The number of terms for the velocity potential expanded as Eq. (1), $N$, is two, to consider both the nonlinearity, and the dispersion, of waves. In the two-dimensional propagation computation, with the initial surface profile given by Eq. (2), the initial velocity potential is zero 


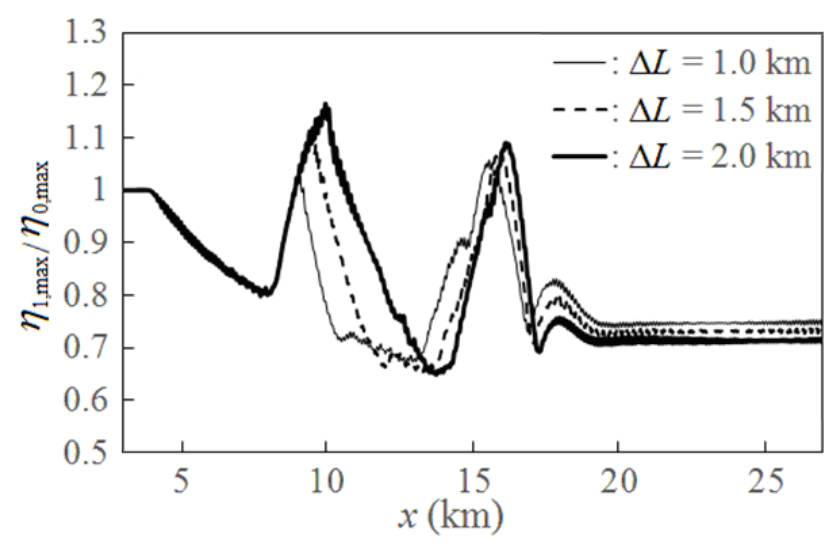

(a) $\Delta L=1.0 \mathrm{~km}, 1.5 \mathrm{~km}$, and $2.0 \mathrm{~km}$

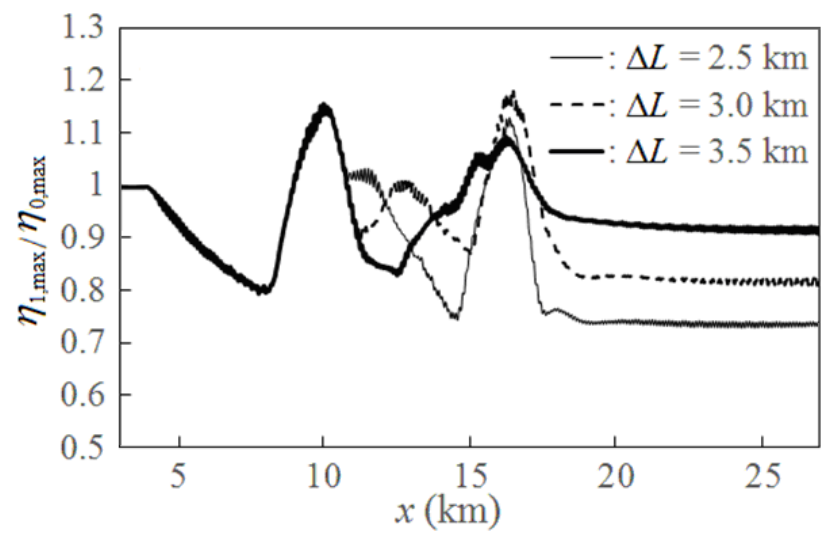

(b) $\Delta L=2.5 \mathrm{~km}, 3.0 \mathrm{~km}$, and $3.5 \mathrm{~km}$

Figure 7. A relative maximum surface level $\eta_{1, \max } / \eta_{0, \max }$, for different values of the distance between two VLFSs, $\Delta L$, where the initial tsunami height $a$ is $5.0 \mathrm{~m}$. The flexural rigidity, and the length, of both VLFSs are $1.0 \times 10^{11} \mathrm{Nm}^{2}$, and $4.0 \mathrm{~km}$, respectively. The offshore end of the offshore-side VLFS, is located at $x=4.0 \mathrm{~km}$. The still water depth is $50.0 \mathrm{~m}$.

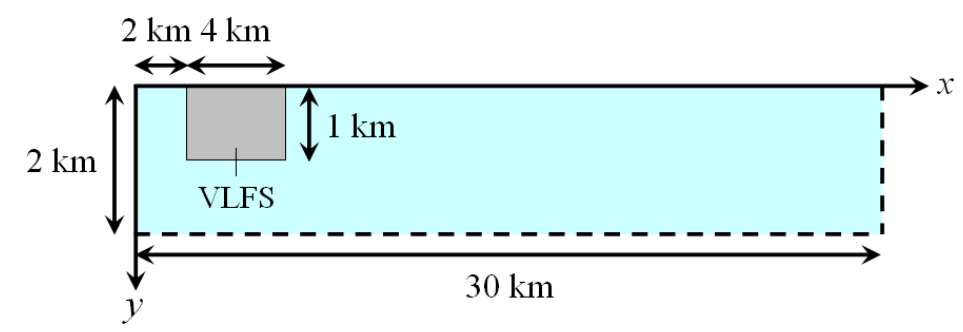

Figure 8. The numerical computation domain for the two-dimensional propagation of a tsunami, interacting with a VLFS, where the VLFS covers the area where $2.0 \mathrm{~km} \leq x \leq 6.0 \mathrm{~km}$ and $0.0 \mathrm{~km} \leq y \leq 1.0 \mathrm{~km}$. The lateral boundary condition, along both $x=0.0 \mathrm{~km}$, and $y=0.0 \mathrm{~km}$, is the reflection condition, while that along both $x=30.0 \mathrm{~km}$, and $y=2.0 \mathrm{~km}$, is the transmission condition.

everywhere.

Shown in Fig. 9 is the time variation of the surface profile, where $\eta$ denotes surface displacement, and the VLFS flexural rigidity $B$ is $1.0 \times 10^{12} \mathrm{Nm}$, in the three dimensions. The tsunami shows disintegration caused by the VLFS, leading to a creation of shorter floating-body waves. Conversely, another wave peak appears, around $x=2.0 \mathrm{~km}$ and $y=1.0 \mathrm{~km}$ when $t=95.0 \mathrm{~s}$, after which it moves to around $x=4.0 \mathrm{~km}$ and $y=1.3 \mathrm{~km}$ when $t=185.0 \mathrm{~s}$. This wave propagates to the outside, along the crest line of the main wave, such that the tsunami height decreases also owing to this outgoing wave, which is not generated in the one-dimensional tsunami propagation. 


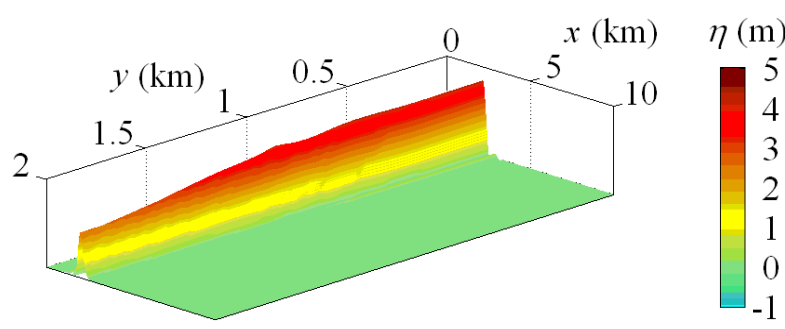

(a) $t=95.0 \mathrm{~s}$

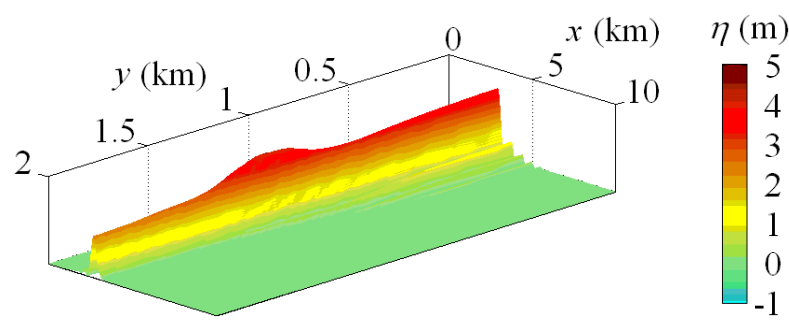

(b) $t=125.0 \mathrm{~s}$

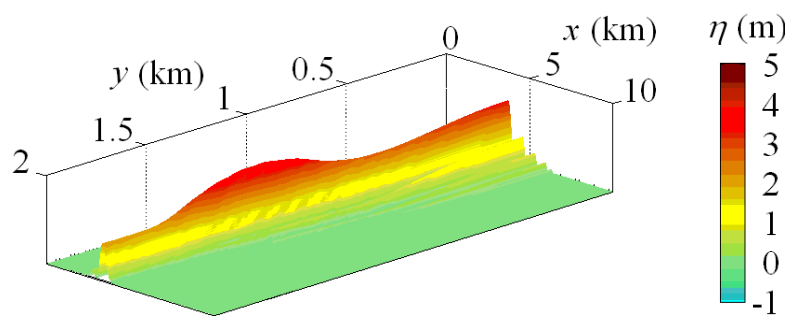

(c) $t=155.0 \mathrm{~s}$

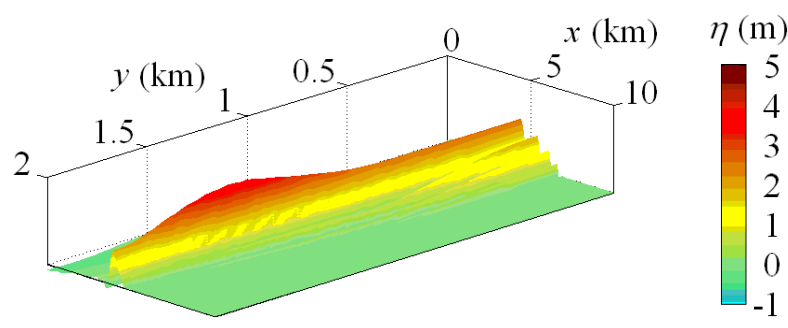

(d) $t=185.0 \mathrm{~s}$

Figure 9. The time variation of the surface profile, where $\eta$ denotes surface displacement; the initial wave height $a$, and the initial wavelength $\lambda$, of the incident tsunami are $5.0 \mathrm{~m}$, and $1.0 \mathrm{~km}$, respectively. The VLFS, the flexural rigidity of which, $B$, is $1.0 \times 10^{12} \mathrm{Nm}^{2}$, covers the area indicated in Fig. 8. The still water depth is $50.0 \mathrm{~m}$.

\section{CONCLUSIONS}

The tsunami-height reduction using a very large floating structure, i.e., a VLFS, or two VLFSs, was discussed, with the water waves, interacting with one, or two, floating thin-plates, respectively, simulated numerically. First, the tsunami height decreased, because the tsunamis showed disintegration, leading to a creation of shorter floating-body waves with larger phase velocities. Second, after passing the VLFS, the main wave overlapped the shorter waves, in the uncovered area, such that the tsunami height increased. Third, the tsunami height decreased again, as the main wave left the shorter waves behind.

The final tsunami-height reduction rate increased, as the length, or the flexural rigidity, of the VLFS was increased. When the VLFS flexural rigidity is larger, it took a longer time for the main wave, to pass a greater number of generated shorter waves, with its slower phase velocity, resulting in a longer time until the main wave obtained the final tsunami-height reduction rate. The final tsunami-height 
reduction rate also increased, as the wave height of the incident tsunami increased, such that the final tsunami-height reduction rate depended on the distance between two VLFSs, concerning the tsunami height at the entrance into the area covered with the onshore-side VLFS. In the two-dimensional tsunami propagation, another wave propagated to the outside, along the crest line of the main wave, leading to an additional tsunami-height reduction.

\section{REFERENCES}

Hermans, A. J. 2000. A boundary element method for the interaction of free-surface waves with a very large floating flexible platform, Journal of Fluids and Structures, 14, 943-956.

Kakinuma, T. 2001. A set of fully nonlinear equations for surface and internal gravity waves, Proceedings of $5^{\text {th }}$ International Conference on Computer Modelling of Seas and Coastal Regions, WIT Press, 225-234.

Kakinuma, T., K. Yamashita, and K. Nakayama. 2012. Surface and internal waves due to a moving load on a very large floating structure, Journal of Applied Mathematics, 2012, 830530, 14p.

Kashiwagi, M. 2004. Transient responses of a VLFS during landing and take-off of an airplane, Journal of Marine Science and Technology, 9, 14-23.

Nakayama, K. and T. Kakinuma. 2010. Internal waves in a two-layer system using fully nonlinear internal-wave equations, International Journal of Numerical Methods in Fluids, 62, 574-590.

Sakai, S., X. Liu, M. Sasamoto, and T. Kagesa. 1998. Experimental and numerical study on the hydroelastic behavior of VLFS under tsunami, Proceedings of $2^{\text {nd }}$ International Conference on Hydroelasticity in Marine Technology, RIAM, Kyushu University, 385-391.

Yamashita, K. and T. Kakinuma. 2014. Properties of surface and internal solitary waves, Proceedings of $34^{\text {th }}$ International Conference on Coastal Engineering, waves. 45, $15 \mathrm{p}$. 